\title{
Sleep's Role in Schema Learning and Creative Insights
}

\author{
Simon J. Durrant ${ }^{1,2}$ D J Jennifer M. Johnson ${ }^{2,3}$ \\ Accepted: 12 January 2021 / Published online: 23 January 2021 \\ (C) The Author(s) 2021
}

\begin{abstract}
Purpose of Review A recent resurgence of interest in schema theory has influenced research on sleep-dependent memory consolidation and led to a new understanding of how schemata might be activated during sleep and play a role in the reorganisation of memories. This review is aimed at synthesising recent findings into a coherent narrative and draw overall conclusions.

Recent Findings Rapid consolidation of schematic memories has been shown to benefit from an interval containing sleep. These memories have shown reduced reliance on the hippocampus following consolidation in both humans and rodents. Using a variety of methodologies, notably including the DRM paradigm, it has been shown that activation of a schema can increase the rate of false memory as a result of activation of semantic associates during slow wave sleep (SWS). Memories making use of a schema have shown increased activity in the medial prefrontal cortex, which may reflect both the schematic activation itself and a cognitive control component selecting an appropriate schema to use. SWS seems to be involved in assimilation of new memories within existing semantic frameworks and in making memories more explicit, while REM sleep may be more associated with creating entirely novel associations while keeping memories implicit.

Summary Sleep plays an important role in schematic memory consolidation, with more rapid consolidation, reduced hippocampal involvement, and increased prefrontal involvement as the key characteristics. Both SWS and REM sleep may have a role to play.
\end{abstract}

Keywords Memory $\cdot$ Schema $\cdot$ Sleep $\cdot$ Hippocampus $\cdot \mathrm{mPFC} \cdot$ Creative thinking

\section{Introduction}

Over the past two decades, renewed research interest has uncovered a wealth of evidence around the role of sleep in memory consolidation - the offline process by which memories are stabilised, reorganised, and in some cases strengthened over time [1•]. Models of consolidation, such as the multiple trace model [2] and the active systems hypothesis [3], have been adapted to account for the role of sleep [4]. However, not all

This article is part of the Topical Collection on Sleep and Learning

Simon J. Durrant

SiDurrant@lincoln.ac.uk

1 School of Psychology, University of Lincoln, Brayford Pool, Lincoln LN6 7TS, UK

2 Lincoln Sleep Research Centre, University of Lincoln, Brayford Pool, Lincoln LN6 7TS, UK

3 School of Health and Social Care, University of Lincoln, Brayford Pool, Lincoln LN6 7TS, UK memories are made equal. The traditional subdivision between declarative and non-declarative memory systems [5] has revealed differences in the way sleep processes these memories and which stages of sleep are most closely involved. More recently, the concept of the memory schema has seen a resurgence of interest [6••] and this has raised the question of just how schematic memories are reprocessed during sleep.

Schema research, which can trace its origins back to the early twentieth century [7], reached an initial peak of interest in the early 1980 s in the wake of the cognitive revolution in psychology, with seminal works looking at schemata as the building blocks of cognition [8], as a central focus of language comprehension [9] and as episodic knowledge structures [10•]. Following a somewhat quieter period, schemata became the focus of renewed interest in the late 2000s, with the publication of an important study on schema learning and memory consolidation in rats $[11 \bullet \bullet]$ and the subsequent controversy over the nature of that consolidation $[12,13]$. From early on, it was identified that schemata play a key role in memory reconstruction, which may not always be entirely accurate or 
faithful to the original memory trace, and that they themselves are constantly open to modification from new insights [14]. Alongside the question of how sleep and schemata may work together in consolidating memory is therefore the question of sleep's role in creative insight and the development of new associations which may necessitate updating or revising a schema. These questions have been the focus of a lot of research in the last decade especially. Just 15 years ago, we knew very little about how consolidation might act on memories that are facilitated by a schema. As recently as 10 years ago - after the initial research findings in rats had suggested more rapid consolidation of schema-conformant memorieswe did not know if this applied in humans, or the neural substrate of this rapid action, or the role of sleep in this process. In this review, we will outline the most salient findings from recent research and highlight what is, and equally importantly what is not, known at the present time.

The concept of a schema has been redefined a number of times throughout the last century of intermittent use. Different researchers have emphasised different aspects depending on their own particular research interest, notably Piaget's [15] introduction of the schema into developmental psychology, Bartlett's [14] emphasis on the adaptability of the schema, and Mandler's [10 ${ }^{\circ}$ focus on the narrative structure of many schemata. Ghosh and Gilboa [6••], reviewing the history of schema research, have identified four features which they believe to be essential: (a) an associative network structure; (b) development across multiple episodes; (c) lack of specific unit/instance detail; (d) adaptability. Durrant et al. [16••] emphasised two additional features in relation to how schemas operate: (e) a schema is an existing cognitive structure (developed on the basis of experience) which is triggered/activated; it is not created by the experience which invokes it; and (f) a schema generally creates a set of expectations as to what will subsequently be encountered. While these definitions are helpful, it is clear that the concept of schema is rather nebulous and open to shifts in emphasis and interpretation which may hinder attempts to understand how the schema affects sleepdependent memory consolidation. This can be alleviated by researchers clearly outlining their intended definition of a schema when describing their research design. We therefore adopt a definition of a schema here which emphasises four key features:

1. A schema is an associative mental structure acquired on the basis of experience. This experience is typically longterm, ranging from days to years, and is derived from many overlapping exemplars.

2. A schema is triggered by a specific context, but the content of the schema - once triggered - is independent of the context. That is to say, a tonal music schema could be triggered by a scale, a chord, or even a whole piece of music, but the schema is the same for each type of trigger.
3. A schema creates a set of expectations. Entering a restaurant may trigger a restaurant schema, for example, which may include an expectation of seeing tables with place settings, being greeted by a waiter or handed a menu. Seeing these items will not be surprising. Entering a clothes shop will trigger a different schema; in that case, being handed a menu would be highly surprising.

4. A schema facilitates rapid processing of new experiences which trigger the schema. This could include earlier or more complete integration into existing semantic networks, better retention of memories, or reduced susceptibility to interference.

\section{Sleep-Dependent Schematic Memory Consolidation}

A key aspect of schematic memory is a behavioural performance benefit - memories that fit well within a schema are remembered better than other memories. This benefit is true not only for encoding or retrieval but also for sleep-dependent consolidation, with evidence of more effective consolidation on the first post-encoding night of schema-conformant items leading to stronger recognition, but with no such benefit for items which did not fit the schema [16*0]. Similarly, individuals who have a schema (such as for navigating 3D virtual environments) showed a benefit of a nap while those without such prior experience did not [17]; more generally, prior knowledge of the domain seems to be essential for this type of consolidation [18]. In language learning, both semantic (meaning) and phonological (sound) information-which may each have their own schema- have shown a benefit of this schema across a night of sleep [19], with lexical competition research suggesting that phonology may consolidate more rapidly [20], while integration into an existing semantic network may be associated with sleep spindles [21] and slow oscillations [22]. Semantic congruence may be enough to recover from the detrimental effect of sleep deprivation at encoding, with subsequent sleep showing stronger consolidation of congruent items [23]. Rapid schematic consolidation may at first appear to contradict the complementary learning systems argument for the need for rapid hippocampal storage followed by slower neocortical integration to avoid catastrophic interference [24]; however, recent simulations suggest that schema-conformant memories can be rapidly integrated within this framework [25•].

The Deese-Roediger-McDermott (DRM) task [26] has been a particularly fruitful source of information about how associative networks, which underpin schemata, are consolidated during sleep. This involves presenting lists of words at encoding which are semantically associated through a keyword (the critical lure) which itself is not included in the list. 
At recall or recognition, memory for the words in the list, but also false memory for the critical lure, is measured. The DRM task has been used a number of times to test the effect of sleepdependent consolidation on veridical and false memory, with results not always consistent. Sleep deprivation at retrieval was shown to enhance false memories [27-29], an effect that was abolished by caffeine [30], suggesting an adenosinergic mechanism (due to the role of caffeine in blocking adenosine receptors and temporarily reducing the effect of homeostatic sleep pressure) and therefore also likely to be alleviated by slow wave activity in normal sleep (which also targets homeostatic sleep pressure). This is consistent with the finding that slow wave sleep (SWS) can decrease false and even veridical memories [31]. However, this study also found that sleeping soon after learning enhanced veridical and false memories, suggesting that sleep may also have a beneficial role for both types of memory in some circumstances; this was also seen in another DRM study where the memory benefit was associated with stronger hippocampal activation at retrieval [32]. Similarly, Payne et al. [33] found a beneficial effect of sleep for veridical and false memories, but that SWS was more detrimental for veridical memories while preserving false memories. This selective benefit for false memories after sleep compared to wake was associated with spindles in the right hemisphere [34], although spindles as a whole were seen to reduce false memories in adolescent girls (but not boys) in another study [35]. An explanation for these disparate results is suggested by Diekelmann et al. [36•], who found enhanced false recall after both sleep and sleep deprivation in comparison to daytime wake and suggested two mechanisms are at work: (1) overnight reorganisation which can lead to an increase in false memories; and (2) a cognitive control component which is undermined by sleep deprivation. Both of these will lead to an increase in false memories, the former through activation of memory associates and the latter through suboptimal schema activation or suppression. A meta-analysis of DRM and sleep found that length of the word list (and therefore size of the associative network) and the choice of testing methodology (recall vs recognition) can also make a difference [37].

\section{The Neural Substrate of Schemata}

Studies using the DRM paradigm and others looking at rapid integration of semantic information when activating a schema have focused on the role of SWS, which is considered the most important for memory reorganisation [38]. Theoretical models have also tended to focus on this, including the iOtA model [39•] which describes how schema-conformant memories can be rapidly assimilated (incorporated into existing networks) during SWS and how overlapping memories can modify or even lead to the creation of new schemata. Ohki and
Takei [40] propose a theoretical model in which sleep spindles and hippocampal ripples are involved in schema activation, assimilation of new information, and accommodation (in which the schema is modified), with these being coordinated by delta oscillations. The hippocampus is important to sleepdependent memory consolidation [41] and evidence suggests that hippocampal involvement in a memory trace decreases as a result of consolidation and neocortical integration [42, 43]. In a seminal study in rats, Tse et al. [11••] demonstrated not only that memories consolidated much more rapidly when using a schema (for a learned environment) but also that hippocampal involvement - necessary to initially develop a schema [44] — was much lower for such memories. Sleep was not specifically investigated in that study, but in humans it has been shown that an environmental schema can be developed during sleep based on semantic regional information [45] and that in an object-location task, once a schema has been developed, new associations are learned more effectively [46]. Importantly, the latter study also showed that while the hippocampus was involved in learning new items, the medial prefrontal cortex (mPFC) was activated only when the schema was invoked.

The roles of the mPFC and the hippocampus during sleep have been shown in a number of studies. Semantic assimilation of new words, which benefits from sleep [47], was accompanied by greater activation in the neocortex for words that had been learned the previous day and greater activation in the hippocampus for novel words [48]. Strong evidence for the specific role of the mPFC comes from a study in which mice developed an environmental schema and demonstrated this by showing sensitivity to information which conflicted with this schema [49]. However, when the mPFC was pharmacogenetically suppressed, this sensitivity to schema violations was reduced, implicating the mPFC in schema activation or regulation. Because of the evidence of hippocampal-based reactivation during SWS, investigations of schema processing during sleep have tended to focus on SWS, but other stages of NREM sleep may also play a role. In a face-location task, greater connectivity between the fusiform gyrus and the mPFC during NREM sleep (stages 1 and 2) was associated with consolidation of schematic memories subsequently better retrieved, while fusiform-hippocampal connectivity (during NREM stage 1) was associated with better encoding of new memories after sleep [50]. Neither SWS nor REM sleep was measured in this study due to technical limitations, so it is possible that they may also play a role, but this study highlights a possible role for other sleep stages.

Given memory reactivation in the hippocampus during SWS and the possible role of the mPFC in selecting and activating a schema to facilitate memory processing during sleep, it is important to first elucidate the relationship between these two structures in the context of schema theory in order to then understand how sleep may govern that relationship. The 
possible roles of the mPFC and hippocampus have been described in the SLIMM (schema-linked interactions between medial prefrontal and medial temporal regions) model of van Kesteren and colleagues [51••]. Essentially, this model proposes that new memories which are congruent with an existing schema will resonate and activate the $\mathrm{MPFC}$, which will in turn suppress the medial temporal lobe (MTL, including the hippocampus). New memories which are incongruent will not resonate, the mPFC will remain silent, and the MTL will bind together the neocortical areas involved. In the case of a partial match with an existing schema, both MPFC and MTL activation may be seen; the extent to which the MPFC and hippocampus cooperate or compete may depend on the extent of the resonance therefore [52]. Related similar ideas have been proposed by other researchers, including Preston et al. [53] who have suggested that the hippocampus is involved in cortical binding while the PFC is involved in creating, selecting, or updating the schema, and Ghosh et al. [6・•] who have suggested that the $\mathrm{mPFC}$ is involved in selecting/ activating task-relevant schemata on a continuous basis. Studies of patients with vmPFC damage show mixed effects; on the one hand, patients who showed confabulation were poorer at rejecting critical lures [54], suggesting a deficit of the cognitive control component, while on the other hand, healthy controls demonstrated more critical lures in the DRM task [55], suggesting the active use of a schema. The $\mathrm{mPFC}$ might thus be performing both functions outlined by Diekelmann et al. [36•].

Evidence in support of this model comes from a variety of studies. When interfering with a movie schema by scrambling the order of part of it, activation of the MPFC was seen for schema-related knowledge and more hippocampal involvement for information difficult to integrate due to the interference [56]. In tasks involving congruency (either multisensory or object-scene visual pairs), with more congruent stimuli believed to more strongly invoke a schema, greater $\mathrm{mPFC}$ and less MTL involvement was seen at encoding [57] and retrieval [58], while the benefit of a schema for associative memory was seen immediately after encoding but for item memory was seen only after a consolidation interval (containing sleep and wake) [59]. Better performance by 2 nd-year biology undergraduates for facts related rather than unrelated to their previous knowledge was also directly associated with higher MPFC activation and lower MTL activation at encoding [60]. Inhibiting activation in the $\mathrm{mPFC}$ prior to encoding through TMS might be expected to inhibit schematic memory processing, and indeed it was found to inhibit production of critical lures in the DRM task, but not veridical memory, at immediate retrieval [61]. In an emotional false memory task following mood induction, the same TMS inhibition had an impact on negative false memory, but not veridical memory, the morning after an overnight consolidation interval [62].
This evidence all points to increased involvement of the $\mathrm{mPFC}$ when a schema is used to help encoding or retrieval together with a decreased role for the hippocampus. What happens during consolidation, and in particular during sleep, has been much less extensively investigated, but some studies have directly examined this in humans. A fact learning task leading to the development of new schemata prior to testing schema-conformant vs non-conformant knowledge showed that increased spindle density during sleep directly predicted both schematic memory consolidation and the hippocampal disengagement for those schematic memories in this task, while other memories continued to involve the hippocampus $[63 \bullet]$.

\section{REM Sleep and Slow Wave Sleep in Schematic Memory Consolidation}

The previous studies point to the role of NREM sleep - and especially SWS - in schema activation and consolidation of schema-conformant memories. Does REM sleep play any role here? A DRM study using emotional as well as neutral words found that both negative and neutral false words were enhanced by sleep [64]; sleep stages were not measured but negative memory is generally considered enhanced by REM sleep $[65,66]$. Similarly, cueing words to be semantically integrated during SWS suggested subsequent processing during REM sleep [67]. More direct evidence comes from a study utilising a strongly established pre-existing musical schema, and stimuli that either matched or did not match this, which showed the benefit of sleep when consolidating schemaconformant items only [16*0]. However, in this case, the schema benefit was associated with frontal and central theta power during REM sleep; this finding suggests that MPFC involvement may extend beyond SWS for certain types of memory, although frontal theta power during SWS has also been seen to benefit targeted memory reactivation when using a tonal music schema [68]. The involvement of REM sleep in schema processing suggests prefrontal activation during REM sleep. Previous reports have suggested that dorsolateral prefrontal activation may diminish during REM sleep [69]; however, this does not apply specifically to the medial prefrontal cortex, for which firing rates during REM sleep remain at a high level [70], suggesting potential schema-related activation during that sleep stage is plausible. Two animal studies are also instructive here. First, $\operatorname{Arc}$ and zif268 genes were shown to be upregulated during associative pair learning in rats, and zif 268 in particular was upregulated more strongly in the mPFC for rats that were able to use a location schema. The expression of immediate early genes including specifically zif 268 is upregulated during REM sleep rather than NREM sleep and in particular in response to prior experience while awake [71]. Furthermore, optogenetically suppressing the $\mathrm{mPFC}$ in mice 
led not only to reduced activation (and zif268 expression) in the $\mathrm{mPFC}$ but also in the hippocampus during encoding, suggesting that the mPFC may directly influence hippocampal activation at encoding [72]. The mPFC may tag items for consolidation using theta activation [73, 74], with evidence from rats showing increased mPFC-hippocampal theta coherence after learning and prefrontal reactivation during SWS [75]. Connectivity between the MPFC and MTL is highly attenuated during REM sleep, so schema-related hippocampal suppression by the mPFC is likely to be specific to SWS [70].

Taken collectively, the evidence suggests that SWS and REM may work together to reprocess schematic memories offline during sleep, according to what was tagged during encoding. The specific roles of REM and SWS remain to be elucidated but some theoretical models have been proposed. Landmann and colleagues [76•, 77] have suggested that SWS is specifically involved in schema formation and integration of new memories, while schema disintegration (in order to restructure them) takes place during REM sleep. A recent study of gist learning and visual statistical learning provided some tentative evidence in support of the latter proposition, but this remains in need of confirmation [78]. A related idea is that SWS may take the lead in stabilising memories while REM sleep is primarily concerned with creatively reorganising them [79]. The key mechanisms through which this may occur are hippocampal input to the neocortex and reactivation during SWS, meaning that gist abstraction of essential overlapping elements takes place during SWS, while during REM sleep, random activation triggered by $\mathrm{PGO}$ waves combined with high levels of acetylcholine encourages the formation of new connections (conceptually and synaptically) [80•].

\section{Creative Thinking and Sleep}

These suggested roles put REM sleep at the centre of creative and inductive thinking, where "creativity involves forming associative elements in new combinations by providing mediating connective links" (Mednick, [81]; 226). Creative thinking is essential for problem-solving and the potential role of sleep has attracted the attention of a number of researchers (see Table 1 for a summary of recent findings). An early demonstration using an anagram task showed that participants woken during REM rather than NREM sleep were better suited to solving the task when asked to do so immediately [82]. Performance on the Remote Associates Task (RAT) - a common measure of creative thinking - has also shown a benefit of REM sleep [83], and in one study both REM and NREM sleep [84], especially for difficult problems [85]. As well as being strongly predicted by the amount of REM sleep, autonomic arousal - measured by heart rate variability and strongest during REM sleep - is also a good predictor of RAT performance [86]. The breadth of association for emotional words primed in advance similarly benefits from REM sleep [87] and REM sleep may also benefit creative problemsolving if metacognitive knowledge (concerning how to go about the task) is given in advance [88]. Interestingly, individuals with narcolepsy-who obtain more and earlier REM sleep-have shown a stronger creative profile compared to healthy controls when evaluated with the Test of Creative Profile and the Creativity Achievement Questionnaire and when creative performance was measured using the Evaluation of Potential Creativity test battery [89]. Furthermore, symptoms of narcolepsy associated with the increase in REM sleep (including more sleep paralysis, more lucid dreaming and more REM sleep behaviour disorder) were associated with higher creativity scores.

However, not all of the available evidence fits with this theory. Using the Abbreviated Torrance Test for Adults to measure creative thinking, NREM was found to be associated with both originality and figural creativity [90] and SWS specifically seemed to benefit problem-solving in a video game task [91]. Sleep of any form was not seen to benefit performance in the Compound Remote Associates task [92] where it strengthened only veridical memory. Research using reactivation has been similarly ambivalent, with creative problemsolving in the Unusual Uses Task benefitting from covert odour-based reactivation throughout the night [93], while creative puzzle-solving showed a benefit when reactivated by playing an associated sound during SWS [94].

The SWS benefit for creative puzzle-solving is also seen in the Number Reduction Task (NRT). A seminal paper from Wagner and colleagues [[95•]; see also [96, 97] for further commentary] showed that the hidden rule embedded in the NRT was discovered by around $60 \%$ of participants in a post-sleep session after first learning the task before sleep, compared to around $20 \%$ after an equivalent wake period or engaging in their first session in either the morning or the evening. This demonstrates both that sleep may play a role in insight based on inductive/ creative thinking and that prior exposure is necessary for this to take place post-sleep. A subsequent study revealed that implicit knowledge of the hidden rule is more likely to become explicit after SWS, while insight is equally likely to develop after either SWS- or REM-rich sleep [98], suggesting that REM sleep may be associated with enhancement of knowledge that remains implicit [99]. The transfer from implicit to explicit knowledge during SWS is associated with alpha power $(8-12 \mathrm{~Hz})$ [100] and topographic reorganisation of slow cortical potentials involving the hippocampus [101]. It is perhaps notable that older adults, with significantly less SWS [102], do not show the same benefit of sleep on the NRT as younger adults [103].

Transfer from implicit to explicit knowledge also occurs in the Serial Reaction Time Task (SRTT) following a period of sleep [104•], something which may be dependent on 
Table 1 Tasks measuring creative thinking or the development of new explicit awareness and the reported role of sleep

\begin{tabular}{|c|c|c|}
\hline Source & Task & Sleep benefit \\
\hline Walker et al. (2002) [1] & Anagram Task & REM sleep \\
\hline Cai et al. (2009) [2] & Remote Associates Task & REM sleep \\
\hline Carlsson, Davidson, and Ors (2019) [3] & Remote Associates Task & REM and NREM sleep \\
\hline $\begin{array}{l}\text { Sio, Monaghan, and Ormerod (2013) } \\
\text { [4] }\end{array}$ & Remote Associates Task & Sleep (stage not measured) \\
\hline Whitehurst et al. (2016) [5] & Remote Associates Task & REM sleep \\
\hline Carr and Nielsen (2015) [6••] & Emotional Word Association & REM sleep \\
\hline Brand et al. (2010) [7] & $\begin{array}{l}\text { Metacognitive knowledge used in the Tower of Hanoi, } \\
\text { Hobbits and Orcs problem, and Katona Card problem }\end{array}$ & REM sleep \\
\hline Lacaux et al. (2019) [8] & $\begin{array}{l}\text { Test of Creative Profile and the Creativity Achievement } \\
\text { Questionnaire }\end{array}$ & $\begin{array}{l}\text { REM sleep (not measured, but implied by narcolepsy } \\
\text { REM characteristics) }\end{array}$ \\
\hline Drago et al. (2011) [9] & Abbreviated Torrance Test & NREM stage 1 and SWS \\
\hline Beijamini et al. (2014) [10•] & Speedy Eggbert Mania ${ }^{\circledR}$ video game & SWS \\
\hline Landmann et al. (2016) [11••] & Compound Remote Associate task & None \\
\hline Ritter et al. (2012) [12] & Unusual Uses Task & Sleep (odour-based reactivation throughout sleep) \\
\hline Sanders et al. (2019) [13] & Rebus, matchstick, spatial, and verbal puzzles & SWS (sound-based reactivation) \\
\hline $\begin{array}{l}\text { Wagner et al. (2004); Yordanova et al. } \\
\qquad(2008,2009,2012)[14,15,16 \bullet \bullet, 17]\end{array}$ & Number Reduction Task & $\begin{array}{l}\text { SWS (for insight to become explicit); SWS and } \\
\text { REM sleep (for insight to develop but remain } \\
\text { implicit) }\end{array}$ \\
\hline Debarnot et al. (2017) [18] & Number Reduction Task in older adults & None \\
\hline Fischer at al (2006) [19] & Serial Reaction Time Task Generation Task & $\begin{array}{l}\text { Sleep (but no associations found with any particular } \\
\text { stage) }\end{array}$ \\
\hline Yordanova et al. (2017) [20] & $\begin{array}{l}\text { Serial Reaction Time Task } \\
\text { Generation Task }\end{array}$ & $\begin{array}{l}\text { Right hemisphere spindles in NREM Stage } 2 \text { and } \\
\text { SWS }\end{array}$ \\
\hline $\begin{array}{l}\text { Drosopoulos, Harrer and Born (2011) } \\
\text { [21] }\end{array}$ & Serial Reaction Time Task Generation Task & Sleep (vs deprivation; stage not measured) \\
\hline Wilhelm et al. (2013) [22] & $\begin{array}{l}\text { Serial Reaction Time Task Generation Task in adults } \\
\text { and children }\end{array}$ & Slow wave activity during NREM sleep \\
\hline Pace-Schott et al. (2012) [23] & Explicit knowledge in the Iowa Gambling Task & Sleep (stage not measured) \\
\hline Zander et al. (2017) [24] & Semantic Coherence Task & Sleep (stage measured but no associations reported) \\
\hline
\end{tabular}

hippocampal involvement [41] and related to NREM spindles [105] but for which explicit instruction of the presence of a sequence is not necessary [106]. Children have more SWS than adults and sure enough this post-sleep explicit awareness is seen more strongly in children [107]. Post-sleep explicit knowledge of information learned implicitly across multiple trials is also seen in the Iowa Gambling task [108] and a semantic coherence task [109]. The benefit of SWS for abstracting underlying probabilities across trials is also seen in statistical learning [110] and associated with a decrease in hippocampal involvement over time [111, 112], although externally cued reactivation during SWS may interfere with this [113]. Explicit awareness of sequence fragments could occur during statistical learning and would explain the role of SWS. By contrast, the probabilities learned in the weather prediction task are likely to remain implicit and REM sleep has been implicated in this task [114]. Underlying grammatical rule abstraction similarly benefits from sleep in both adults [115•] and children [116, 117] and may benefit from SWS in particular [118].

\section{Conclusions}

The research described in this review is a broad body of work focused on schematic memory processing and the crucial role of sleep in this. It is apparent that while not all research findings are in complete agreement, there are some clear trends in the findings and an overall broadly coherent narrative considering the complex and diverse nature of schemata and the wide variety of techniques used to probe schematic memory processing during sleep. This allows us to draw a number of key conclusions:

1. Memories which trigger a schema are generally remembered better and consolidated quicker.

2. A schema will involve additional associates being activated and may result in an increase in false memory.

3. Cognitive control of schema selection and the activation of the schema itself are two distinct processes, both likely to involve the mPFC. The former is undermined by preencoding sleep deprivation while the latter benefits from post-encoding sleep. 

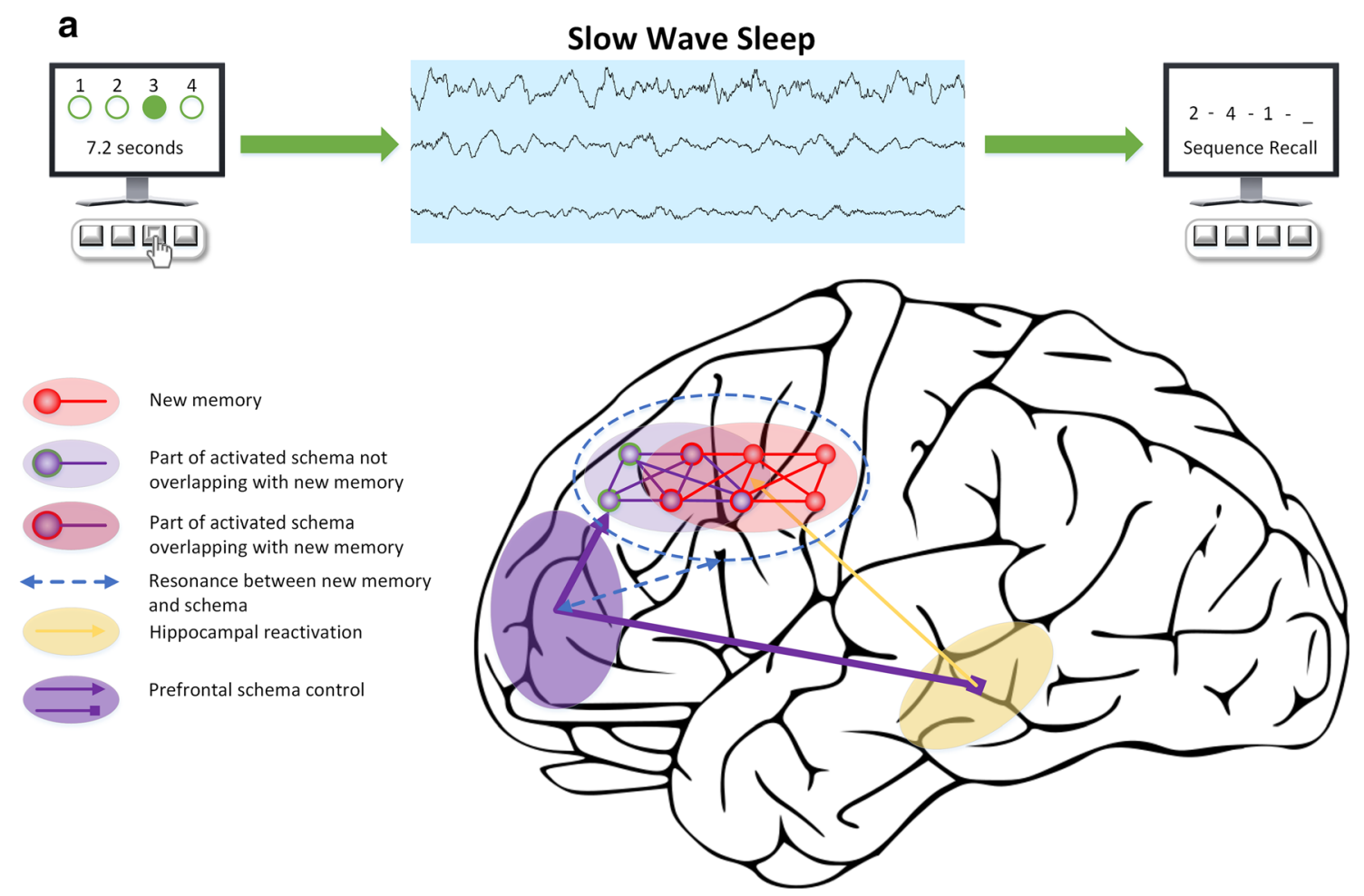

b
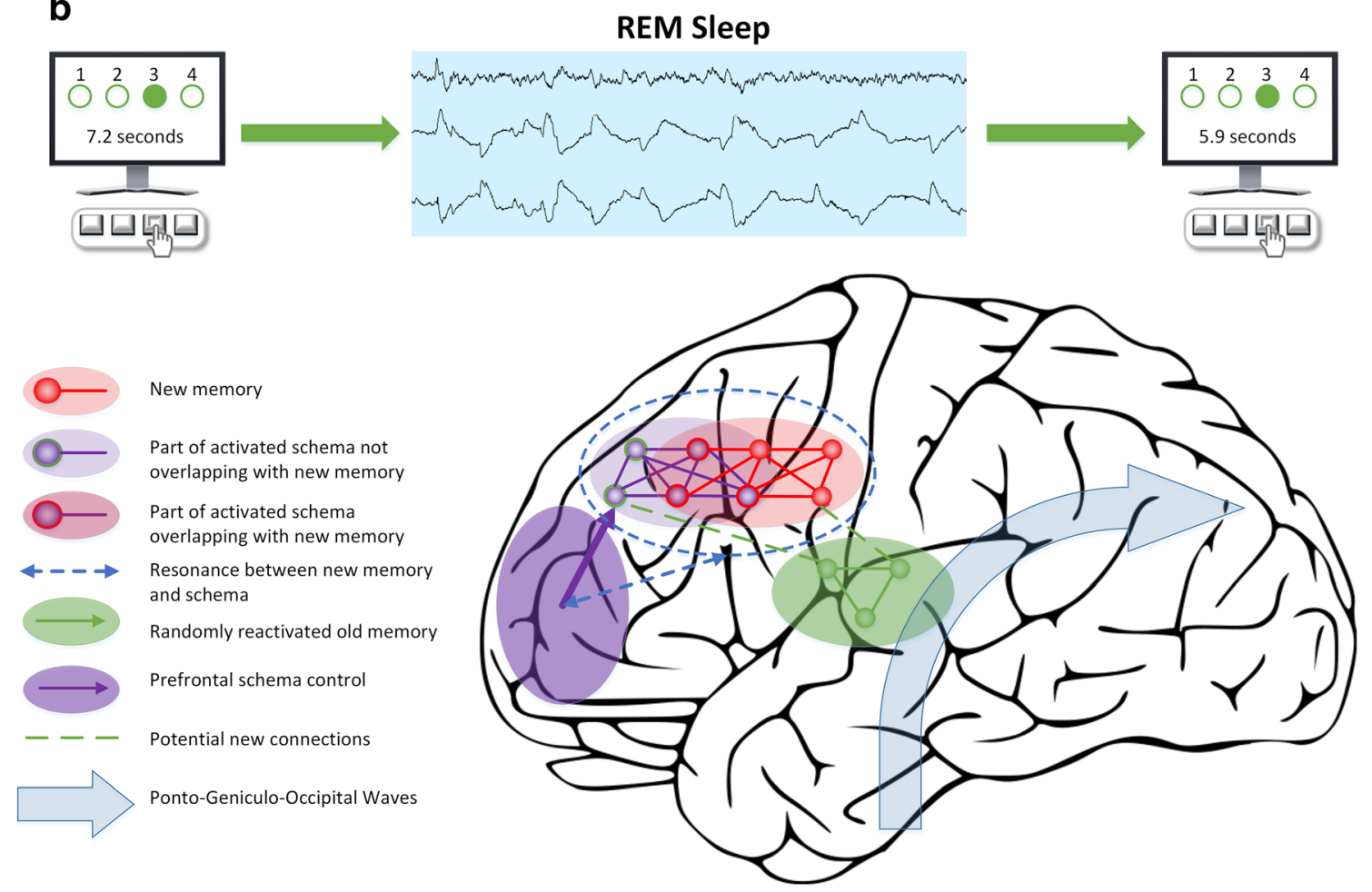

Fig. 1 New memory reactivation, schema usage and behavioural consequences in the context of (A) SWS and (B) REM sleep. See text for details

4. Where a memory is integrated rapidly into a schema or existing semantic network, hippocampal involvement will decrease in proportion to the schema involvement and speed of consolidation. The hippocampus may be directly suppressed by the
$\mathrm{mPFC}$ to prevent it from reactivating and reorganising memories which already fit into an existing framework.

5. Schema activation and assimilation of a new memory within an existing schema seem most likely to happen 
during SWS, though more evidence is needed for this and REM sleep may also play a role.

6. Schematic accommodation-where a schema is significantly modified to account for a new memory - may involve both SWS to modify existing connections where necessary and REM sleep to incorporate new connections to previously unrelated knowledge.

7. Creative thinking - forming of new associations - may benefit from SWS where significant existing knowledge is required for the activity or may benefit from REM sleep where novelty is required. Most likely, cycles involving both SWS (to activate existing associated knowledge) and REM sleep (to allow encourage formation of new and relatively distantly related associations) may be needed.

8. SWS is important for making implicit knowledge explicit, while REM sleep may be important for consolidating implicit knowledge while keeping it implicit.

Some of these findings are drawn together and illustrated in Fig. 1:

A. In Panel A, a new memory (red) is initially reactivated by the hippocampus (yellow) during SWS. This memory resonates (blue dashed surrounding line) with an existing schema, and this resonance is monitored (blue dashed straight line) by the prefrontal schema control component (dark purple) which fully activates the schema (light purple) and also suppresses the hippocampus. Part of that schema overlaps with the memory (purple circles with red edges) and part of it does not (purple circles with green edges). The part that does not overlap but which is activated anyway may appear as false memory in subsequent recall. The increased activation of associates may lead to insight based on prior experience and the initial hippocampal involvement could allow this insight to become an explicit memory.

B. In Panel B, a new memory (red) is reactivated during REM sleep. Similar to SWS, this memory may again resonate (blue dashed surrounding line) with an existing schema which is then fully activated by the schema control component (dark purple). At the same time, PGO waves cause the activation of a less closely related or even entirely unrelated memory (green). The coincident activation of the two together with early gene expression encouraging plasticity causes new and perhaps unexpected connections to be formed (green dashed lines) between the new memory and the old one, leading to creative thinking and new associations. The reactivation also strengthens the new memory and binds it to the schema, but in the absence of medial temporal lobe activation, this leads to stronger implicit memory performance rather than becoming explicit.
These conclusions represent our best understanding given the existing evidence to date. We know much more than we did 10 years ago: in the last decade, we have established that rapid consolidation of schema-conformant memories occurs in humans that it involves greater activation in the MPFC and reduced activation in the hippocampus and that both SWS and REM sleep may have a role to play in this process. However, more evidence is still needed in all of these areas. Existing studies tend to be based on small samples, using different methodologies, different participants, different paradigms of sleep manipulation and monitoring, and different forms of data analysis, yielding results which do not always sit easily together. Patterns are starting to emerge and we are beginning to understand the important role of schemata in memory processing and how sleep is involved in this, but a lot remains to be discovered about the specific neurobiological mechanisms involved, the circumstances in which different aspects of sleep are involved, and how these relate to specific behavioural outcomes. This field remains open and active.

\section{Compliance with Ethical Standards}

Conflict of Interest The authors declare that they have no conflict of interest.

Human and Animal Rights and Informed Consent This article does not contain any studies with human or animal subjects performed by any of the authors.

Open Access This article is licensed under a Creative Commons Attribution 4.0 International License, which permits use, sharing, adaptation, distribution and reproduction in any medium or format, as long as you give appropriate credit to the original author(s) and the source, provide a link to the Creative Commons licence, and indicate if changes were made. The images or other third party material in this article are included in the article's Creative Commons licence, unless indicated otherwise in a credit line to the material. If material is not included in the article's Creative Commons licence and your intended use is not permitted by statutory regulation or exceeds the permitted use, you will need to obtain permission directly from the copyright holder. To view a copy of this licence, visit http://creativecommons.org/licenses/by/4.0/.

\section{References}

Papers of particular interest, published recently, have been highlighted as:

- Of importance

-• Of major importance

1. Rasch B, Born J. About sleep's role in memory. Physiol Rev. 2013;93:681-766 A comprehensive overview of the evidence surrounding sleep-dependent memory consolidation.

2. Nadel L, Moscovitch M. Memory consolidation, retrograde amnesia and the hippocampal complex. Curr Opin Neurobiol. 1997;7:217-27. 
3. Frankland PW, Bontempi B. The organization of recent and remote memories. Nat Rev Neurosci. 2005;6:119-30.

4. Born J, Wilhelm I. System consolidation of memory during sleep. Psychol Res. 2012;76:192-203.

5. Tulving E. Episodic and semantic memory. Organ Mem. 1972;1: 381-403.

6.• •- Ghosh VE, Gilboa A. What is a memory schema? A historical perspective on current neuroscience literature. Neuropsychologia. 2014;53:104-14 A clear overview of the history of schema theory to the present time and listing many of the essential features of a schema.

7. Head BYH, Holmes G. Sensory disturbances from cerebral lesions. Brain. 1911;34:103-254.

8. Rumelhart DE. Schemata: the building blocks of cognition. In: Bruce BC, Brewer WF, editors. Spiro RJ. Hillside: Theor. issues Read. Compr. Erlbaum; 1980. p. 33-58.

9. Anderson RC. Role of the reader's schema in comprehension, learning, and memory. In: Anderson R, Osborn J, Tierney R, editors. Theor. Model. Process. Read. 4th ed. Newark: International Reading Association; 1984. p. 594-606.

10. - Mandler JM. Stories, scripts, and scenes: aspects of schema theory. Hillsdale: Erlbaum; 1984. A detailed overview of schema theory as seen in the 1980s but including essential features of modern definitions

11.. .. Tse D, Langston RF, Kakeyama M, Bethus I, Spooner PA, Wood ER, et al. Schemas and memory consolidation. Science (80- ). 2007;316:76-82 Very important study of schematic memory consolidation in rats and the role of the hippocampus, which has inspired a lot of the subsequent work in this area.

12. Tse D, Langston RF, Bethus I, Wood ER, Witter MP, Morris RGM. Does assimilation into schemas involve systems or cellular consolidation? It's not just time. Neurobiol Learn Mem. 2008;89: $361-5$.

13. Squire LR. Rapid consolidation. Science (80- ). 2007;316:57-8.

14. Bartlett FC. Remembering : a study in experimental and social psychology. Cambridge: Social Psychology; 1932. p. 1-11.

15. Piaget J. The origins of intelligence in children. New York: International Universities Press; 1952.

16.• Durrant SJ, Cairney SA, McDermott C, Lewis PA. Schemaconformant memories are preferentially consolidated during REM sleep. Neurobiol Learn Mem. 2015;122:41-50. https://doi. org/10.1016/j.nlm.2015.02.011 The first study to look directly at neural responses, sleep, and schematic memory consolidation in humans. The study used a robust and welldefined schema and included overnight polysomnography.

17. Wamsley EJ, Tucker MA, Payne JD, Stickgold R. A brief nap is beneficial for human route-learning: the role of navigation experience and EEG spectral power. Learn Mem. 2010;17:332-6.

18. Groch S, Schreiner T, Rasch B, Huber R, Wilhelm I. Prior knowledge is essential for the beneficial effect of targeted memory reactivation during sleep. Sci Rep. 2017;7:39763. https://doi.org/10. 1038/srep39763.

19. Havas V, Taylor JSH, Vaquero L, de Diego-Balaguer R, Rodríguez-Fornells A, Davis MH. Semantic and phonological schema influence spoken word learning and overnight consolidation. Q J Exp Psychol. 2018;71:1469-81.

20. Gaskell MG, Dumay N. Lexical competition and the acquisition of novel words. Cognition. 2003;89:105-32.

21. Tamminen J, Payne JD, Stickgold R, Wamsley EJ, Gaskell MG. Sleep spindle activity is associated with the integration of new memories and existing knowledge. J Neurosci. 2010;30:1435660 .

22. Tamminen J, Lambon Ralph MA, Lewis PA. The role of sleep spindles and slow-wave activity in integrating new information in semantic memory. J Neurosci. 2013;33:15376-81.
23. Alberca-Reina E, Cantero JL, Atienza M. Semantic congruence reverses effects of sleep restriction on associative encoding. Neurobiol Learn Mem. 2014;110:27-34.

24. McClelland JL, McNaughton BL, O'Reilly RC. Why there are complementary learning systems in the hippocampus and neocortex: insights from the successes and failures of connectionist models of learning and memory. Psychol Rev. 1995;102:419-57.

25. McClelland JL. Incorporating rapid neocortical learning of new schema-consistent information into complementary learning systems theory. J Exp Psychol Gen. 2013;142:1190-210 An update of the complementary learning system theory to incorporate rapid schematic memory processing.

26. Roediger HL, McDermott KB. Creating false memories: remembering words not presented in lists. J Exp Psychol Learn Mem Cogn. 1995;21:803-14.

27. Chatburn A, Kohler MJ, Payne JD, Drummond SPA. The effects of sleep restriction and sleep deprivation in producing false memories. Neurobiol Learn Mem. 2017;137:107-13.

28. Verma K, Kashyap N. Sleep deprivation enhances false memory on the Deese-Roediger-McDermott (DRM) task. Psychol Thought. 2019;12:120-30.

29. Lo JC, Chong PLH, Ganesan S, Leong RLF, Chee MWL. Sleep deprivation increases formation of false memory. J Sleep Res. 2016;25:673-82.

30. Diekelmann S, Landolt HP, Lahl O, Born J, Wagner U. Sleep loss produces false memories. PLoS One. 2008;3:e3512. https://doi. org/10.1371/journal.pone.0003512.

31. Pardilla-Delgado E, Payne JD. The impact of sleep on true and false memory across long delays. Neurobiol Learn Mem. 2017;137:123-33.

32. Darsaud A, Dehon H, Lahl O, Sterpenich V, Boly M, Dang-Vu T, et al. Does sleep promote false memories? J Cogn Neurosci. 2011;23:26-40.

33. Payne JD, Schacter DL, Propper RE, Huang LW, Wamsley EJ, Tucker MA, et al. The role of sleep in false memory formation. Neurobiol Learn Mem. 2009;92:327-34.

34. Shaw JJ, Monaghan P. Lateralised sleep spindles relate to false memory generation. Neuropsychologia. 2017;107:60-7.

35. Kuula L, Tamminen J, Makkonen T, Merikanto I, Räikkönen K, Pesonen AK. Higher sleep spindle activity is associated with fewer false memories in adolescent girls. Neurobiol Learn Mem. 2019;157:96-105.

36. • Diekelmann S, Born J, Wagner U. Sleep enhances false memories depending on general memory performance. Behav Brain Res. 2010;208:425-9 An important theoretical contribution proposing separate schema activation and cognitive control components of the prefrontal response during the DRM task.

37. Newbury CR, Monaghan P. When does sleep affect veridical and false memory consolidation? A meta-analysis. Psychon Bull Rev. 2019;26:387-400.

38. Diekelmann S, Born J. Slow-wave sleep takes the leading role in memory reorganization. Nat Rev Neurosci. 2010;11:218.

39. Lewis PA, Durrant SJ. Overlapping memory replay during sleep builds cognitive schemata. Trends Cogn Sci. 2011;15:343-51 The first theoretical model (iOtA) of how schemata might benefit from, and be formed during, slow wave sleep.

40. Ohki T, Takei Y. Neural mechanisms of mental schema: a triplet of delta, low beta/spindle and ripple oscillations. Eur J Neurosci. 2018;48:2416-30.

41. Spencer RMC, Sunm M, Ivry RB. Sleep-dependent consolidation of contextual learning. Curr Biol. 2006;16:1001-5.

42. Takashima A, Nieuwenhuis ILC, Jensen O, Talamini LM, Rijpkema M, Fernández G. Shift from hippocampal to neocortical centered retrieval network with consolidation. J Neurosci. 2009;29:10087-93. 
43. Durrant SJ, Lewis PA. Memory consolidation: tracking transfer with functional connectivity. Curr Biol. 2009;19:R860-2. https:// doi.org/10.1016/j.cub.2009.08.019.

44. McKenzie S, Frank AJ, Kinsky NR, Porter B, Rivière PD, Eichenbaum H. Hippocampal representation of related and opposing memories develop within distinct, hierarchically organized neural schemas. Neuron. 2014;83:202-15.

45. Noack H, Schick W, Mallot H, Born J. Sleep enhances knowledge of routes and regions in spatial environments. Learn Mem. 2017;24:140-4.

46. Van Buuren M, Kroes MCW, Wagner IC, Genzel L, Morris RGM, Fernández G. Initial investigation of the effects of an experimentally learned schema on spatial associative memory in humans. J Neurosci. 2014;34:16662-70.

47. Dumay N, Gaskell MG. Sleep-associated changes in the mental representation of spoken words. Psychol Sci. 2007;18:35-9.

48. Davis MH, Di Betta AM, Macdonald MJE, Gaskell MG. Learning and consolidation of novel spoken words. J Cogn Neurosci. 2009;21:803-20.

49. Richards BA, Xia F, Santoro A, Husse J, Woodin MA, Josselyn SA, et al. Patterns across multiple memories are identified over time. Nat Neurosci. 2014;17:981-6.

50. van Dongen EV, Takashima A, Barth M, Fernández G. Functional connectivity during light sleep is correlated with memory performance for face-location associations. Neuroimage. 2011;57:26270.

51.• van Kesteren MTR, Ruiter DJ, Fernández G, Henson RN. How schema and novelty augment memory formation. Trends Neurosci. 2012;35:211-9 Paper describing the SLIMM model of schematic memory consolidation, which is an important theoretical framework in this area.

52. Gilboa A, Marlatte H. Neurobiology of schemas and schemamediated memory. Trends Cogn Sci. 2017;21:618-31.

53. Preston AR, Eichenbaum H. Interplay of hippocampus and prefrontal cortex in memory. Curr Biol. 2013;23:R764-73.

54. Ghosh VE, Moscovitch M, Colella BM, Gilboa A. Schema representation in patients with ventromedial PFC lesions. J Neurosci. 2014;34:12057-70.

55. Warren DE, Jones SH, Duff MC, Tranel D. False recall is reduced by damage to the ventromedial prefrontal cortex: implications for understanding the neural correlates of schematic memory. J Neurosci. 2014;34:7677-82.

56. Van Kesteren MTR, Fernández G, Norris DG, Hermans EJ. Persistent schema-dependent hippocampal-neocortical connectivity during memory encoding and postencoding rest in humans. Proc Natl Acad Sci U S A. 2010;107:7550-5.

57. Van Kesteren MTR, Beul SF, Takashima A, Henson RN, Ruiter DJ, Fernández G. Differential roles for medial prefrontal and medial temporal cortices in schema-dependent encoding: from congruent to incongruent. Neuropsychologia. 2013;51:2352-9.

58. Van Kesteren MTR, Rijpkema M, Ruiter DJ, Fernández G, Fernandez G, Fernández G, et al. Retrieval of associative information congruent with prior knowledge is related to increased medial prefrontal activity and connectivity. J Neurosci. 2010;30: 15888-94.

59. Van Kesteren MTR, Rijpkema M, Ruiter DJ, Fernández G. Consolidation differentially modulates schema effects on memory for items and associations. PLoS One. 2013;8:e56155.

60. Van Kesteren MTR, Rijpkema M, Ruiter DJ, Morris RGM, Fernández G. Building on prior knowledge: schema-dependent encoding processes relate to academic performance. J Cogn Neurosci. 2014;26:2250-61.

61. Berkers RMWJ, van der Linden M, de Almeida RF, Müller NCJ, Bovy L, Dresler M, et al. Transient medial prefrontal perturbation reduces false memory formation. Cortex. 2017;88:42-52.
62. Bovy L, Berkers RMWJ, Pottkämper JCM, Varatheeswaran R, Fernández G, Tendolkar I, et al. Transcranial magnetic stimulation of the medial prefrontal cortex decreases emotional memory schemas. Cereb Cortex. 2020;30:3608-16.

63. Hennies N, Ralph MAL, Kempkes M, Cousins JN, Lewis PA. Sleep spindle density predicts the effect of prior knowledge on memory consolidation. J Neurosci. 2016;36:3799-810 Study looking directly at sleep and the neural substrate of schematic memory consolidation using polysomnography and fMRI.

64. McKeon S, Pace-Schott EF, Spencer RMC. Interaction of sleep and emotional content on the production of false memories. PLoS One. 2012;7:e49353.

65. Baran B, Pace-Schott EF, Ericson C, Spencer RMC. Processing of emotional reactivity and emotional memory over sleep. J Neurosci. 2012;32:1035-42.

66. Harrington MO, Johnson JM, Croom HE, Pennington K, Durrant SJ. The influence of REM sleep and SWS on emotional memory consolidation in participants reporting depressive symptoms. Cortex. 2018;99:281-95.

67. Tamminen J, Lambon Ralph MA, Lewis PA. Targeted memory reactivation of newly learned words during sleep triggers REMmediated integration of new memories and existing knowledge. Neurobiol Learn Mem. 2017;137:77-82.

68. Gao D, Long S, Yang H, Cheng Y, Guo S, Yu Y. SWS brainwave music may improve the quality of sleep : an EEG study. Front Neurosci. 2020;14:1-7.

69. Muzur A, Pace-Schott EF, Hobson JA. The prefrontal cortex in sleep. Trends Cogn Sci. 2002;6:475-81.

70. Wierzynski CM, Lubenov EV, Gu M, Siapas AG. Statedependent spike-timing relationships between hippocampal and prefrontal circuits during sleep. Neuron. 2009;61:587-96.

71. Ribeiro S, Mello CV, Velho T, Gardner TJ, Jarvis ED, Pavlides C. Induction of hippocampal long-term potentiation during waking leads to increased extrahippocampal zif-268 expression during ensuing rapid-eye-movement sleep. J Neurosci. 2002;22:1091423.

72. Bero AW, Meng J, Cho S, Shen AH, Canter RG, Ericsson M, et al. Early remodeling of the neocortex upon episodic memory encoding. Proc Natl Acad Sci U S A. 2014;111:11852-7.

73. Benchenane K, Tiesinga PH, Battaglia FP. Oscillations in the prefrontal cortex: a gateway to memory and attention. Curr Opin Neurobiol. 2011;21:475-85.

74. Inostroza M, Born J. Sleep for preserving and transforming episodic memory. Annu Rev Neurosci. 2013;36:79-102.

75. Benchenane K, Peyrache A, Khamassi M, Tierney PL, Gioanni Y, Battaglia FP, et al. Coherent theta oscillations and reorganization of spike timing in the hippocampal- prefrontal network upon learning. Neuron. 2010;66:921-36.

76. Landmann N, Kuhn M, Piosczyk H, Feige B, Baglioni C, Spiegelhalder K, et al. The reorganisation of memory during sleep. Sleep Med Rev. 2014;18:1-11 Theoretical paper that outlines possible roles for SWS and REM sleep for schema formation, integration, and disintegration.

77. Landmann N, Kuhn M, Maier JG, Spiegelhalder K, Baglioni C, Frase L, et al. REM sleep and memory reorganization: potential relevance for psychiatry and psychotherapy. Neurobiol Learn Mem. 2015;122:28-40.

78. Matorina N, Poppenk J (2019) Sleep promotes relational overlapping memories for long-term generalization. bioRxiv. https://doi. org/10.1101/578492.

79. Pereira SIR, Lewis PA. The differing roles of NREM and REM sleep in the slow enhancement of skills and schemas. Curr Opin Physiol. 2020;15:82-8.

80. Lewis PA, Knoblich G, Poe G. How memory replay in sleep boosts creative problem-solving. Trends Cogn Sci. 2018;22: 
491-503 Outline of the BiOtA mode, which builds on iOtA by adding a postulated role for REM sleep.

81. Mednick S. The associative basis of the creative process. Psychol Rev. 1962;69:220-32.

82. Walker MP, Liston C, Hobson JA, Stickgold R. Cognitive flexibility across the sleep-wake cycle: REM-sleep enhancement of anagram problem solving. Cogn Brain Res. 2002;14:317-24.

83. Cai DJ, Mednick SA, Harrison EM, Kanady JC, Mednick SA. REM, not incubation, improves creativity by priming associative networks. Proc Natl Acad Sci U S A. 2009;106:10130-4.

84. Carlsson I, Davidson P, Ors M. Effects of a daytime nap on primed and repeated remote associates tests and relations with divergent creativity. Creat Res J. 2019;31:207-14.

85. Sio UN, Monaghan P, Ormerod T. Sleep on it, but only if it is difficult: effects of sleep on problem solving. Mem Cogn. 2013;41:159-66.

86. Whitehurst LN, Cellini N, McDevitt EA, Duggan KA, Mednick SC. Autonomic activity during sleep predicts memory consolidation in humans. Proc Natl Acad Sci U S A. 2016;113:7272-7.

87. Carr M, Nielsen T. Morning REM sleep naps facilitate broad access to emotional semantic networks. Sleep. 2015;38:433-43.

88. Brand S, Opwis K, Hatzinger M, Holsboer-Trachsler E. REM sleep is related to the transfer of implicit procedural knowledge following metacognitive learning. Somnologie. 2010;14:213-20.

89. Lacaux C, Izabelle C, Santantonio G, De Villèle L, Frain J, Lubart $\mathrm{T}$, et al. Increased creative thinking in narcolepsy. Brain. 2019;142:1988-99.

90. Drago V, Foster PS, Heilman KM, Aricò D, Williamson J, Montagna $\mathrm{P}$, et al. Cyclic alternating pattern in sleep and its relationship to creativity. Sleep Med. 2011;12:361-6.

91. Beijamini F, Pereira SIR, Cini FA, Louzada FM. After being challenged by a video game problem, sleep increases the chance to solve it. PLoS One. 2014;9:e84342. https://doi.org/10.1371/ journal.pone.0084342.

92. Landmann N, Kuhn M, Maier JG, Feige B, Spiegelhalder K, Riemann D, et al. Sleep strengthens but does not reorganize memory traces in a verbal creativity task. Sleep. 2016;39:705-13.

93. Ritter SM, Strick M, Bos MW, Van Baaren RB, Dijksterhuis A. Good morning creativity: task reactivation during sleep enhances beneficial effect of sleep on creative performance. J Sleep Res. 2012;21:643-7.

94. Sanders KEG, Osburn S, Paller KA, Beeman M. Targeted memory reactivation during sleep improves next-day problem solving. Psychol Sci. 2019;30:1616-24.

95. Wagner U, Gais S, Haider H, Verleger R, Born J. Sleep inspires insight. Nature. 2004;427:352-5 Seminal paper demonstrating the benefit of sleep for creative insight.

96. Stickgold R, Walker M. To sleep, perchance to gain creative insight? Trends Cogn Sci. 2004;8:191-2.

97. Verleger R, Rose M, Wagner U, Yordanova J, Kolev V. Insights into sleep's role for insight: studies with the number reduction task. Adv Cogn Psychol. 2013;9:160-72.

98. Yordanova J, Kolev V, Verleger R, Bataghva Z, Born J, Wagner U. Shifting from implicit to explicit knowledge: different roles of early- and late-night sleep. Learn Mem. 2008;15:508-15.

99. Smith C. Sleep states and memory processes in humans: procedural versus declarative memory systems. Sleep Med Rev. 2001;5:491-506.

100. Yordanova J, Kolev V, Wagner U, Born J, Verleger R. Increased alpha (8-12 Hz) activity during slow wave sleep as a marker for the transition from implicit knowledge to explicit insight. J Cogn Neurosci. 2012;24:119-32.
101. Yordanova J, Kolev V, Wagner U, Verleger R. Covert reorganization of implicit task representations by slow wave sleep. PLoS One. 2009;4:e5675. https://doi.org/10.1371/journal.pone. 0005675 .

102. Ohayon MM, Carskadon MA, Guilleminault C, Vitiello MV. Meta-analysis of quantitative sleep parameters from childhood to old age in healthy individuals: developing normative sleep values across the human lifespan. Sleep. 2004;27:1255-73.

103. Debarnot U, Rossi M, Faraguna U, Schwartz S, Sebastiani L. Sleep does not facilitate insight in older adults. Neurobiol Learn Mem. 2017;140:106-13.

104. Fischer S, Drosopoulos S, Tsen J, Born J. Implicit learning explicit knowing: a role for sleep in memory system interaction. J Cogn Neurosci. 2006;18:311-9 The first clear demonstration of implicitly learned sequences being made explicit after a period of sleep.

105. Yordanova J, Kolev V, Bruns E, Kirov R, Verleger R. Sleep spindles in the right hemisphere support awareness of regularities and reflect pre-sleep activations. Sleep. 2017;40:1-13.

106. Drosopoulos S, Harrer D, Born J. Sleep and awareness about presence of regularity speed the transition from implicit to explicit knowledge. Biol Psychol. 2011;86:168-73.

107. Wilhelm I, Rose M, Imhof KI, Rasch B, Büchel C, Born J. The sleeping child outplays the adult's capacity to convert implicit into explicit knowledge. Nat Neurosci. 2013;16:391-3.

108. Pace-Schott EF, Nave G, Morgan A, Spencer RMC. Sleepdependent modulation of affectively guided decision-making. J Sleep Res. 2012;21:30-9.

109. Zander T, Volz KG, Born J, Diekelmann S. Sleep increases explicit solutions and reduces intuitive judgments of semantic coherence. Learn Mem. 2017;24:641-5.

110. Durrant SJ, Taylor C, Cairney S, Lewis PA. Sleep-dependent consolidation of statistical learning. Neuropsychologia. 2011;49: 1322-31.

111. Durrant SJ, Cairney SA, Lewis PA. Overnight consolidation aids the transfer of statistical knowledge from the medial temporal lobe to the striatum. Cereb Cortex. 2013;23:2467-78. https://doi.org/ 10.1093/cercor/bhs244.

112. Durrant SJ, Cairney SA, Lewis PA. Cross-modal transfer of statistical information benefits from sleep. Cortex. 2016;78:85-99. https://doi.org/10.1016/j.cortex.2016.02.011.

113. Hennies N, Lambon Ralph MA, Durrant SJ, Cousins JN, Lewis PA. Cued memory reactivation during SWS abolishes the beneficial effect of sleep on abstraction. Sleep. 2017;40:394-406.

114. Djonlagic I, Rosenfeld A, Shohamy D, Myers C, Gluck M, Stickgold R. Sleep enhances category learning. Learn Mem. 2009;16:751-5.

115. Nieuwenhuis ILC, Folia V, Forkstam C, Jensen O, Petersson KM. Sleep promotes the extraction of grammatical rules. PLoS One. 2013;8:e65046. https://doi.org/10.1371/journal.pone.0065046.

116. Gómez RL, Bootzin RR, Nadel L. Naps promote abstraction in language-learning infants. Psychol Sci. 2006;17:670-4 Important early study demonstrating sleep-dependent abstraction in infants.

117. Hupbach A, Gomez RL, Bootzin RR, Nadel L. Nap-dependent learning in infants. Dev Sci. 2009;12:1007-12.

118. Gaskell MG, Warker J, Lindsay S, Frost R, Guest J, Snowdon R, et al. Sleep underpins the plasticity of language production. Psychol Sci. 2014;25:1457-65.

Publisher's Note Springer Nature remains neutral with regard to jurisdictional claims in published maps and institutional affiliations. 